\title{
Penile aseptic abscess in the cavernous body at the base of the penis: a case report
}

Case report

Man-Cheng Xiaa, Ke-Qiang Yina, Yu-Sheng Wang ${ }^{a}$, Jia-Wei Chena, Xiao-Dong Biana, Wei-Bing Shuang,**

${ }^{a}$ First Clinical Medical College, Shanxi Medical University, Taiyuan, Shanxi 030001, China

${ }^{b}$ Department of Urology, First Hospital of Shanxi Medical University, Taiyuan, Shanxi 030001, China

Received: 26 April 2020; Accepted: 11 June 2020; Published: 20 March 2021

\begin{abstract}
We report a case of aseptic abscess in the cavernous body at the base of the penis. In our clinical observation, the patient underwent puncture and drainage of the corpus cavernosum abscess, followed by surgical resection of the abscess wall, with the incisions closed layer by layer with primary suture. In addition, we paid attention to strengthening the postoperative management by using elastic bandages to wrap the penis intermittently to prevent edema; the incision would not be covered with dressings from the third day after the operation, so as to keep the incision site dry in an open way. During the period of indwelling of the catheter after the operation, we noticed the care of the external orifice of the urethra to reduce the occurrence of catheter-related infections. Finally, the patient was diagnosed with a penile aseptic abscess in the cavernous body at the base of the penis. The patient recovered well after surgery and was discharged 1 week later. At 1.5 years after the operation, the shape of the penis returned to normal, and the erectile function was normal. It was seen that good nursing concept is of great help for prognosis, which could avoid infection and edema, and is conducive to wound healing.
\end{abstract}

Keywords: penis $\bullet$ aseptic abscess $\bullet$ puncture and drainage $\bullet$ primary suture $\bullet$ nursing

(c) Shanxi Medical Periodical Press.

\section{Introduction}

Penile abscess is a rare genitourinary disease, which was first reported by Niedrach et al. in 1989. ${ }^{1}$ The pathogens usually include Neisseria gonorrhoeae, Escherichia coli, Staphylococcus aureus, Streptococcus, or mixed bacteria. ${ }^{1-4}$ Treatment of penile abscess mainly includes surgical incision and drainage, puncture and drainage, and antibiotic treatment. ${ }^{3,5-8}$ In the present clinical observation, we report a case of aseptic abscess in the cavernous body at the base of the penis. The patient underwent puncture and drainage of the corpus cavernosum abscess, followed by surgical resection of the abscess wall, with the incisions closed layer by layer with primary suture.

How to cite this article: Xia MC, Yin KQ, Wang YS, Chen JW, Bian XD, Shuang WB. Penile aseptic abscess in the cavernous body at the base of the penis: a case report. Front Nurs. 2021;1:83-90.

* Corresponding author.

E-mail: shuangweibing@126.com (W. -B. Shuang).

○ Open Access. @ 2021 Xia et al., published by Sciendo. (G)BY This work is licensed under the Creative Commons Attribution 4.0 License. 


\section{Case report}

A 65-year-old male patient was admitted to the hospital due to the appearance of a mass at the base of the penis, which was accompanied by dysuria and a thin urine stream. The patient denied any history of penile injection or trauma. Physical examination showed that the mass was located at the base of the penis. The computed tomography (CT) scan and spiral CT 3-D image revealed the presence of a cystic mass measuring $4.9 \times 3.5 \mathrm{~cm}$ in the cavernous body at the base of the penis, which contained a few solid components. The plain CT value was about 13-30 HU (Figures 1 and 2). Both T1-weighted and T2-weighted images confirmed the presence of a round-like cystic mass in the cavernous body, which was surrounded by a clear cystic wall (Figures 3 and 4), and the mass exhibited high signal intensity in diffusion-weighted imaging (DWI) scan (Figure 5), with a clear lesion boundary and a size measuring about $4.2 \mathrm{~cm} \times 4.6 \mathrm{~cm} \times 4.7 \mathrm{~cm}$. The patient's HIV and syphilis tests were negative, and tumor marker levels were within the normal range.

The patient underwent puncture and drainage of the corpus cavernosum abscess, followed by surgical resection of the abscess wall. During the surgical procedure, the skin on the penis and the subcutaneous fascia were resected layer by layer, and a cystic solid mass with a size measuring about $4.9 \mathrm{~cm} \times 3.5 \mathrm{~cm}$ was found under the white membrane. About $40 \mathrm{ml}$ of reddish- brown purulent liquid was aspirated with a syringe, which was confirmed as the lesion and submitted to the laboratory for analysis of bacterial culture (Figure 6). After the abscess cavity was completely opened (Figure 7), the abscess wall was removed and sent for pathological examination. After careful washing with iodophor and saline, a $10-\mathrm{cm}$ scar was found on the right side of the penis; the scar was situated opposite to the cavity in the corpus cavernosum on the opposite side of the tunica albuginea, with no obvious damage to the urethra. The scar was formed possibly due to penile trauma which may be concealed by the patient. Finally, the incisions were closed layer by layer with primary suture. In addition, we strengthened the postoperative management, using elastic bandages to wrap the penis intermittently to prevent edema; the incision would not be covered with dressings from the third day after the operation, so as to keep the incision site dry in an open way. During the period of indwelling of the catheter after the operation, we paid attention to the care of the external orifice of the urethra to reduce the occurrence of catheter-related infections.

Pathological examination showed that suppurative inflammation was accompanied by necrosis and abscess formation. A few inflammatory cells which were not cancer cells were observed on the abscess wall (Figure 8). In addition, no bacteria were found in the pus culture. Finally, the patient was diagnosed with a penile aseptic abscess in the cavernous body at the base of the penis. Ten months after the operation, the

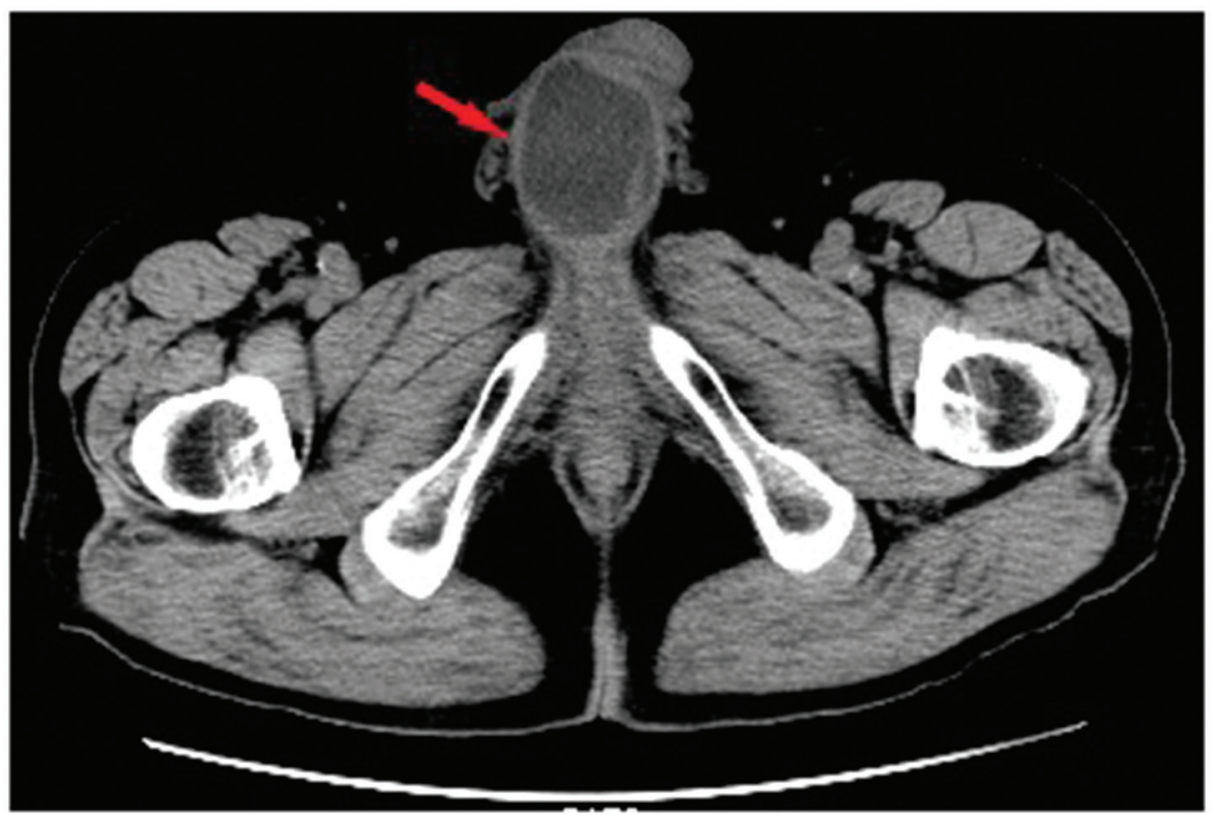

Figure 1. CT scan of a mass of $4.9 \times 3.5 \mathrm{~cm}$ found in the cavernous body of the penis root. 


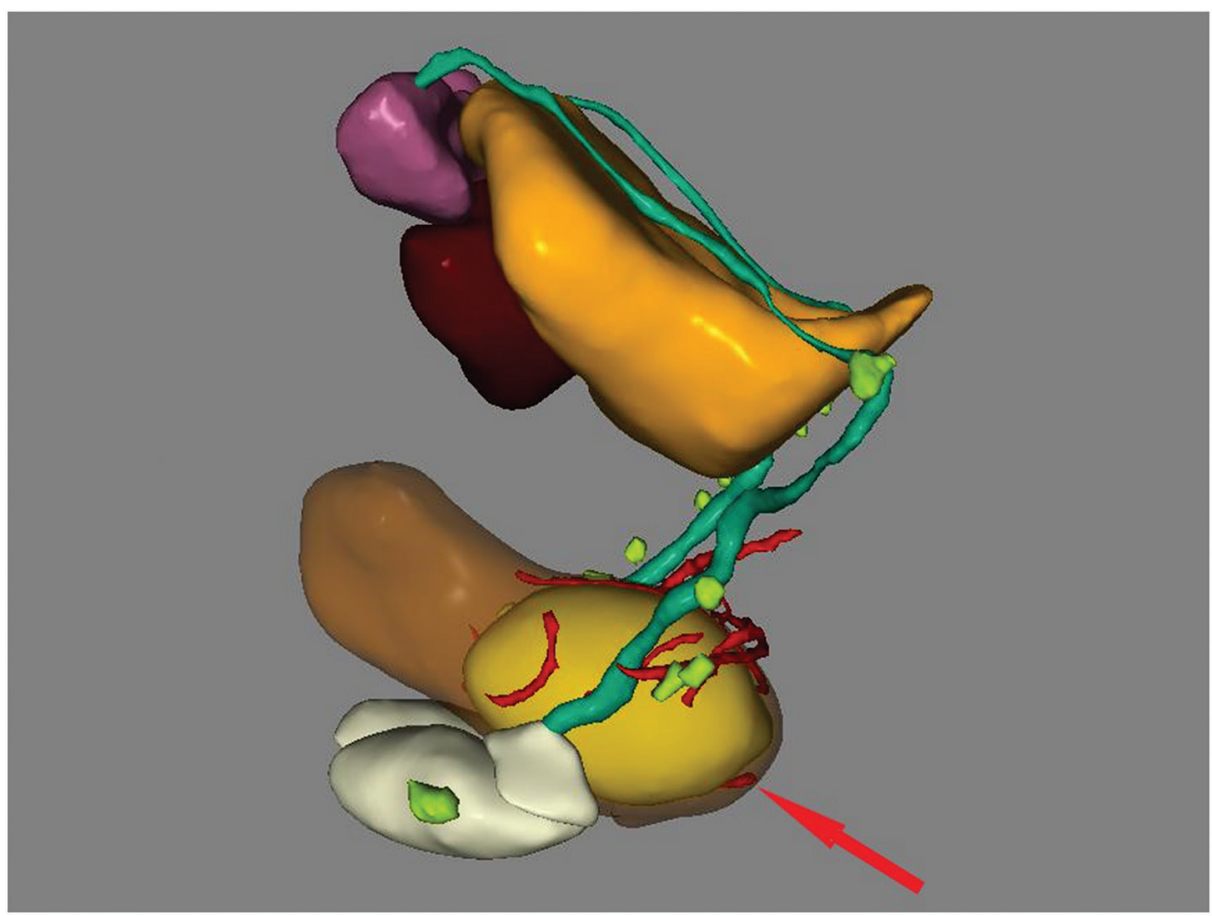

Figure 2. Spiral CT 3-D image of a mass of $4.9 \times 3.5 \mathrm{~cm}$ found in the cavernous body of the penis root.

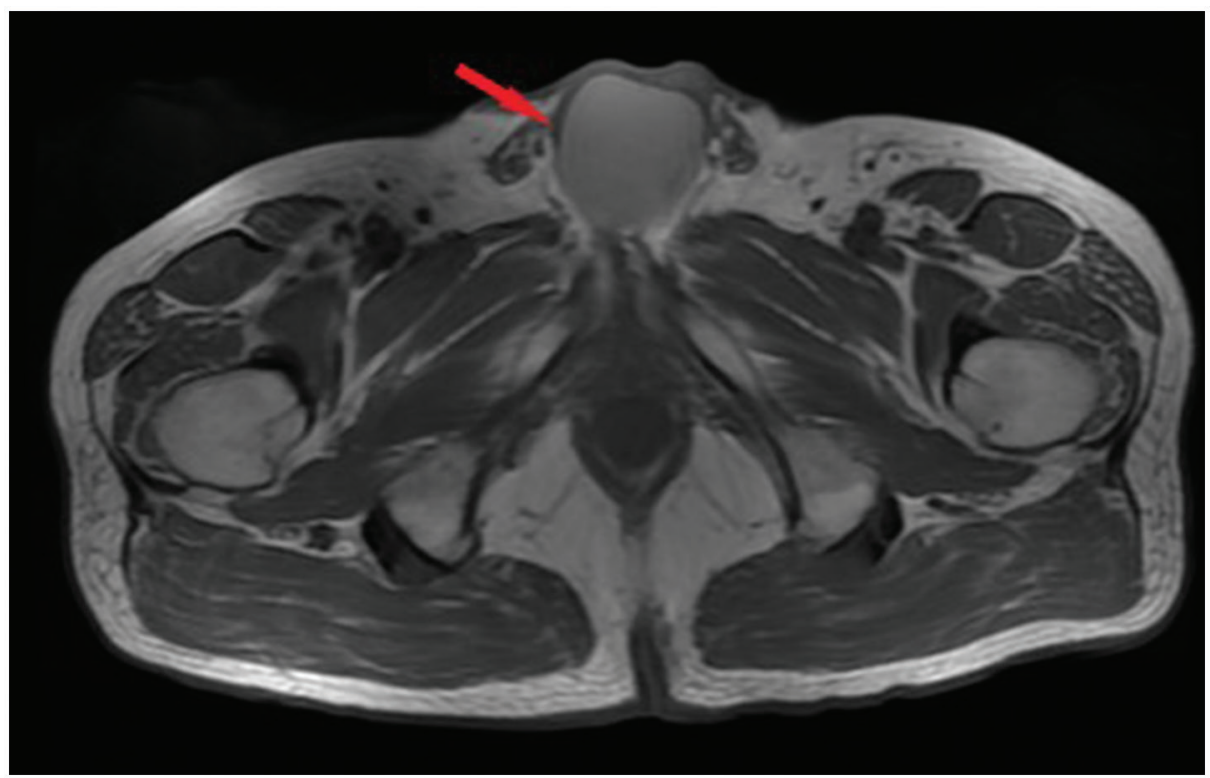

Figure 3. T1-weighted images showing a round-like abscess in the cavernous body of the penis root.

patient recovered well without erectile dysfunction. The patient recovered well after surgery and was discharged 1 week later. At 1.5 years after the operation, the shape of the penis returned to normal, and the erectile function was normal.

\section{Discussion}

The most common symptom of penile abscess is localized penile swelling and painful erections, ${ }^{4}$ but the symptoms in this case included poor urination and a thin 


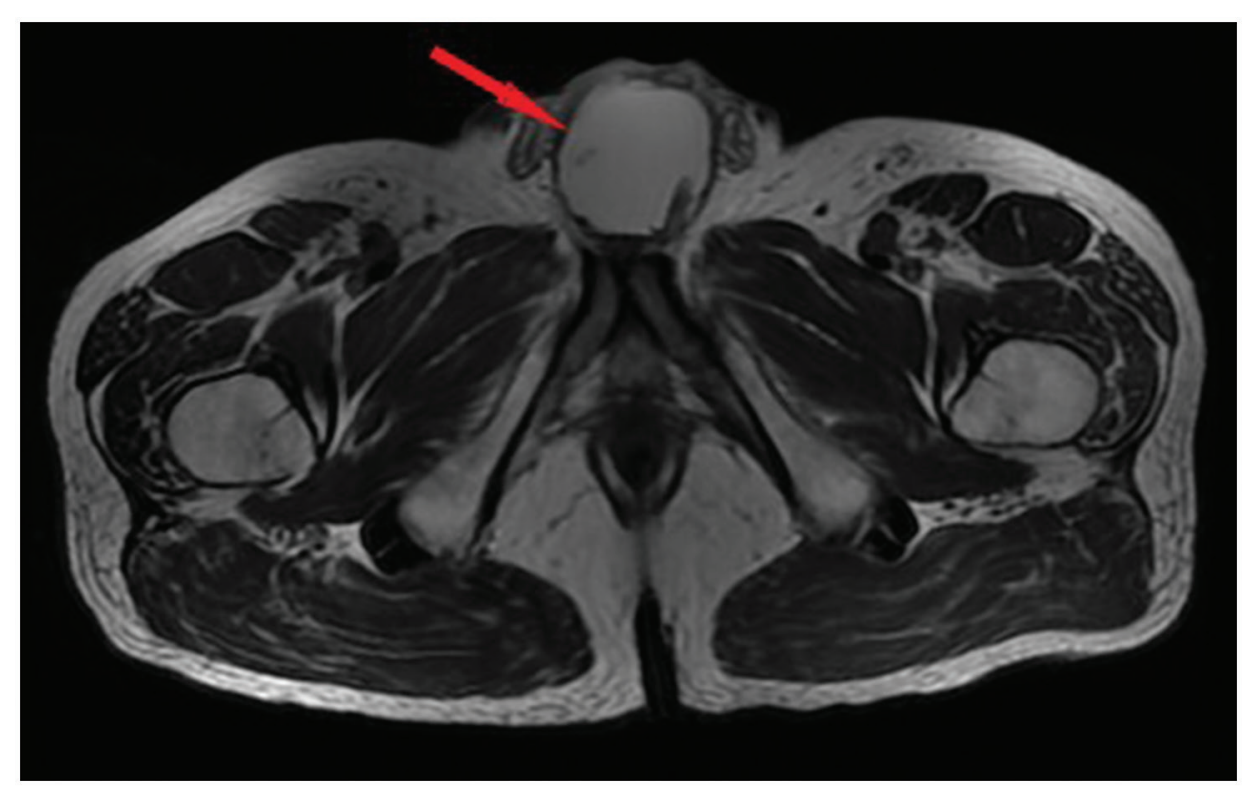

Figure 4. T2-weighted images showing a round-like abscess in the cavernous body of the penis root.

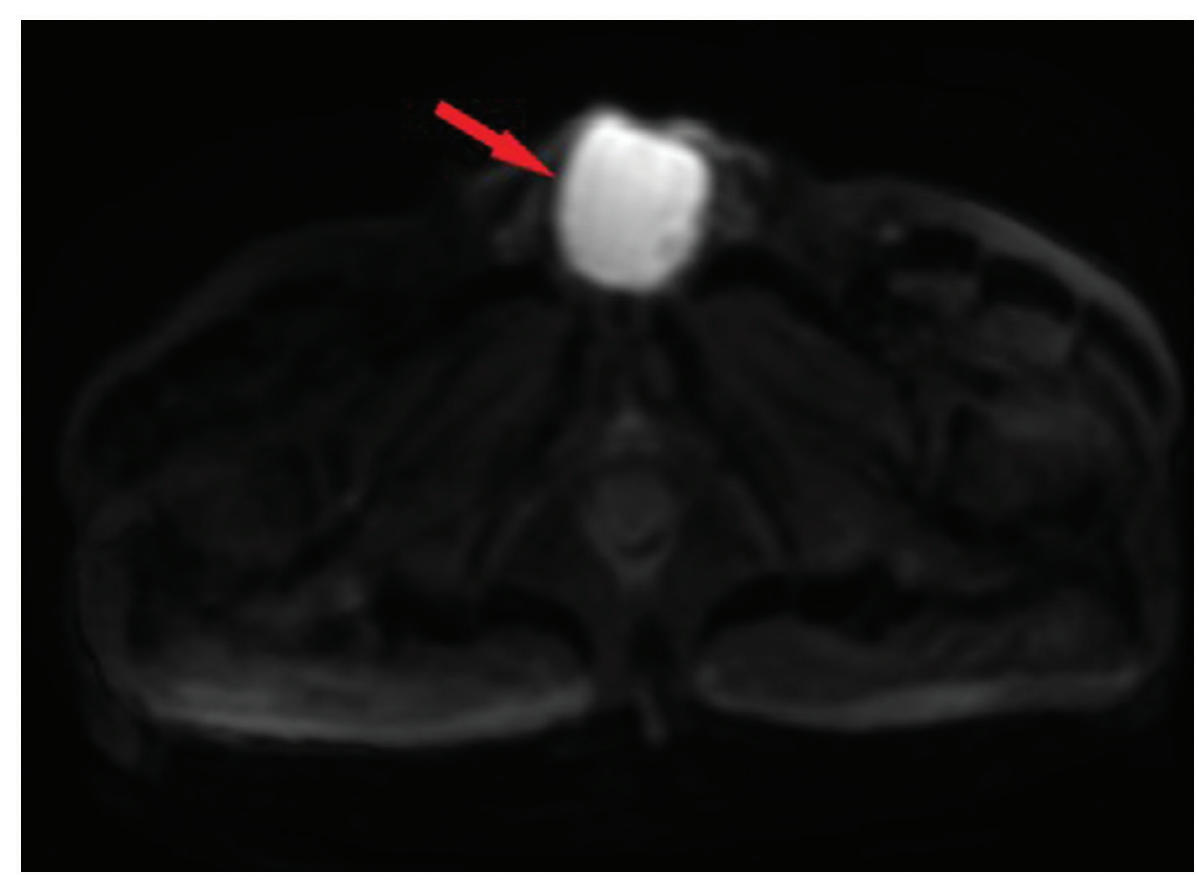

Figure 5. DWI scan showing a round-like cystic mass with high signal intensity in the cavernous body of the penis root.

urine stream. Penile abscess often occurs in the cavernous body, its head, and subcutaneous fascia of the penis, and it is usually caused by a variety of pathogenic bacteria, including N. gonorrhoeae, E. coli, S. aureus, Streptococcus, or mixed bacteria. ${ }^{1-4}$ The formation of a penile abscess may be related to penile trauma, history of urological operation, injection, and disseminated infection. ${ }^{9-11}$ In this case, penile aseptic abscess may be caused by localized bleeding and hematoma from incomplete rupture of the penile tunica albuginea.

Although ultrasound examination is an economical, convenient, and effective method for the diagnosis of penile abscess, ${ }^{6,12} \mathrm{CT}$ and magnetic resonance imaging (MRI) can easily identify cavernous abscess around the 


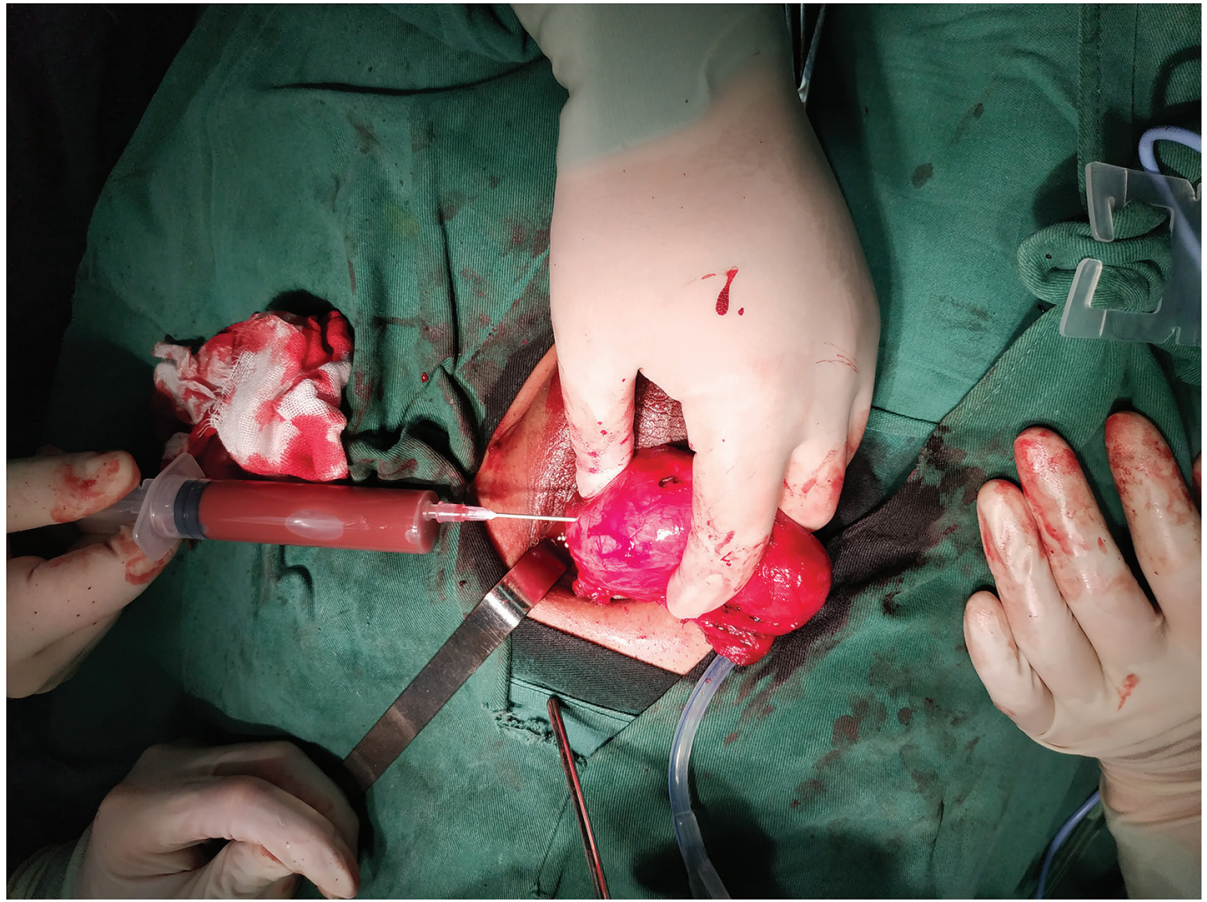

Figure 6. Aspiration of about $40 \mathrm{ml}$ of reddish brown purulent fluid with a syringe.

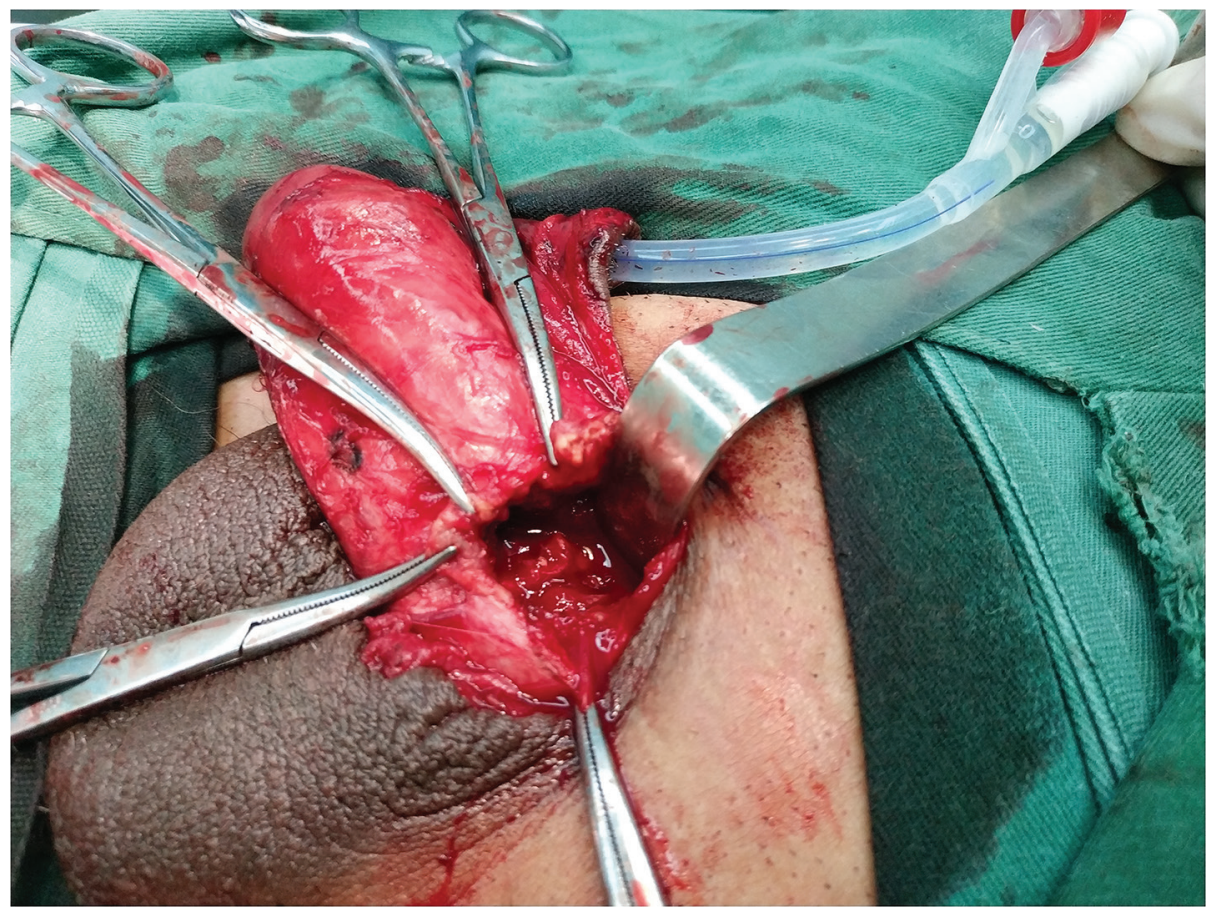

Figure 7. Abscess cavity completely opened during surgery.

root of penile tissue and better reflect its range and relationship with adjacent tissues. ${ }^{4,11} \mathrm{MRI}$, especially DWI, has been considered as a powerful tool for the diagnosis of penile abscess. ${ }^{11}$ Nevertheless, the final diagnosis has to be made based on pathological observation and bacterial culture. 


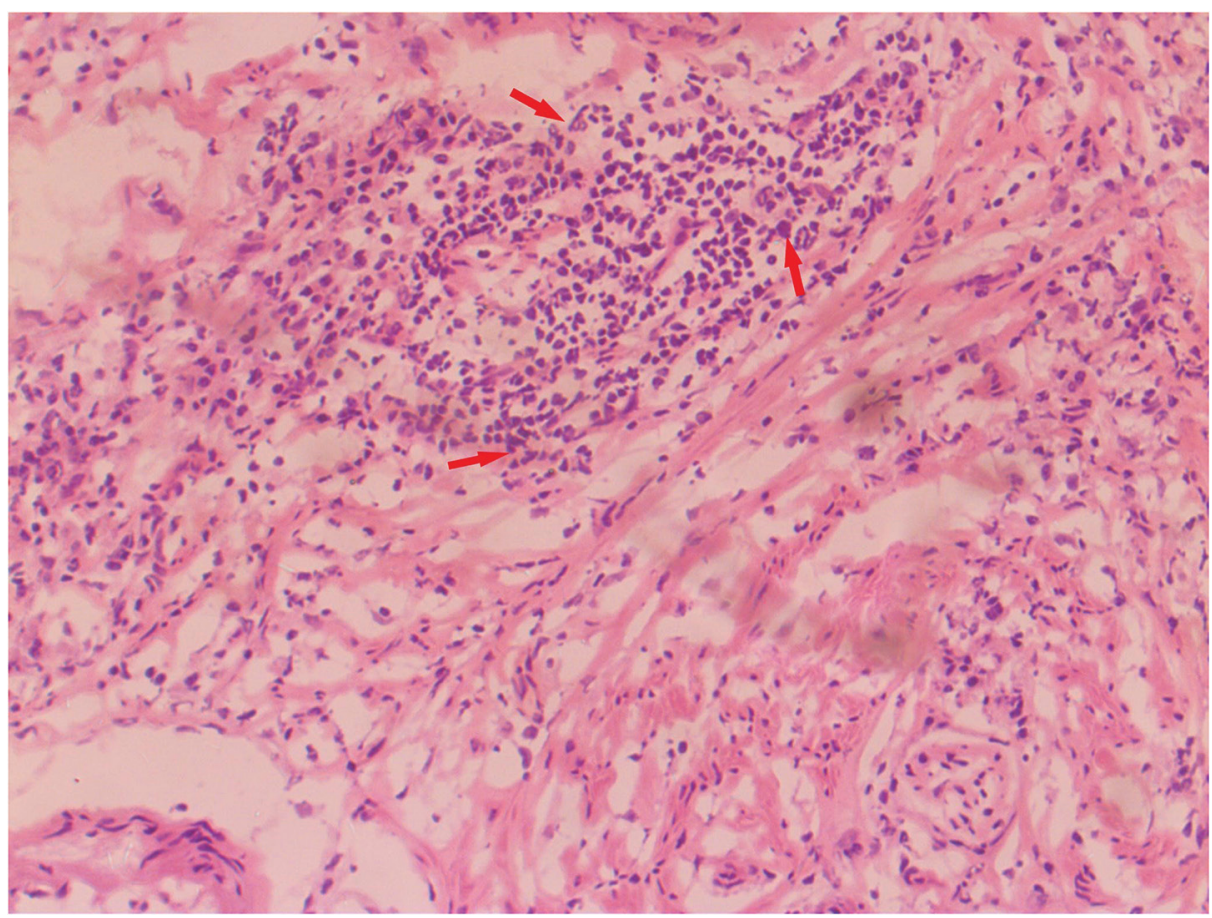

Figure 8. Histopathological examination showing the presence of a few inflammatory cells on the abscess wall.

Treatment of penile abscess mainly includes incision and drainage, puncture and drainage, and antibiotic treatment. ${ }^{3,5-8}$ If the patients experience severe postoperative erectile dysfunction, they need to be further treated with penile prosthesis implantation. ${ }^{13,14}$ Some patients with recurrent penile abscess or pain may have to undergo penectomy. ${ }^{15-17}$ In the present case, not only was the patient treated with puncture drainage, but also the abscess wall was removed and then the gap was closed layer by layer with primary suture. In addition, we took care to strengthen the postoperative management, using elastic bandages to wrap the penis intermittently to prevent edema; the incision would not be covered with dressings from the third day after the operation, so as to keep the incision site dry in an open way. During the period of indwelling of the catheter after the operation, we paid attention to the care of the external orifice of the urethra to reduce the occurrence of catheterrelated infections. Although it was reported that patients who received open surgical drainage are more likely to have complications, including poor erectile function, impaired sexual activity, and secondary fibrosis, $, 0,14,18,19$ this patient recovered well from surgery and had a normal erectile function and appearance of the penis, which may be related to a good nursing concept.

\section{Conclusions}

As a rare disease, the penile aseptic abscess can be diagnosed by a combination of ultrasound, CT, MRI, pathological examination, and bacterial culture. This disease can be effectively treated with surgery for the aspiration of purulent substances using a syringe, followed by surgical resection of the abscess wall. The incisions can also be closed layer by layer with primary suture. During the surgical procedure, rapid discharge of purulent substances is the main therapeutic target. In addition, good nursing concept is of great help for prognosis; this can avoid infection and edema, and is conducive to wound healing. The prognosis of the patient was satisfactory.

\section{Ethical approval}

Ethical issues are not involved in this report.

\section{Conflicts of interest}

All the contributing authors declare that no conflicts of interest exist. 


\section{References}

1. Niedrach WL, Lerner RM, Linke CA. Penile abscess involving the corpus cavernosum: a case report. J Urol. 1989;141:374-375.

2. Nalmas S, Bishburg E, Chan T. Streptococcus constellatus and Prevotella bivia penile abscess. Sci. World J. 2007;7:1631-1633.

3. Brennan J, O'kelly F, Quinlan DM. A case of spontaneous abscess of the corpus cavernosum. Scand J Urol. 2013;47:534-536.

4. Garcia C, Winter M, Chalasani V, Dean T. Penile abscess: a case report and review of literature. Urol Case Reports. 2014;2:17-19.

5. Huuskonen J, Aaltomaa S. Candida sepsis originating from bulbar abscess of the penis. Scand J Urol Nephrol. 2006;40:347-349.

6. Jinga V, Iconaru V. Penile abscess and urethrocutaneous fistula following intracavernous injection: a case report. $J$ Sex Med. 2012;9:3270-3273.

7. Kumabe A, Kenzaka T, Yamamoto Y, Kajii E. Corpus cavernosum abscess from a blind-ending urethra after urinary diversion surgery. BMJ Case Rep. 2013;2013:bcr2013009471.

8. Tüzel E. Spontaneous corpus cavernosum abscess in a healthy man using long-term androgenic anabolic steroids. World J Men's Health. 2015;33:36-38.

9. Al-Reshaid RA, Madbouly K, Al-Jasser A. Penile abscess and necrotizing fasciitis secondary to neglected false penile fracture. Urol Ann. 2010;2:86-88.

10. Song W, Ko KJ, Shin SJ, Ryu DS. Penile abscess secondary to neglected penile fracture after intracavernosal vasoactive drug injection. World J Men's Health. 2012;30:189-191.

11. Yamada K, Horikawa M, Shinmoto H. Magnetic resonance imaging findings of penile abscess. Urology. 2019;131: e5-e6.

12. Palacios A, Massó $P$, Versos R, et al. Penile abscess. Case report. Archivos Esp Urol. 2006;59:809-811 (in Spanish).

13. Dempster NJ, Maitra NU, McAuley L, Brown M, Hendry D. A unique case of penile necrotizing fasciitis secondary to spontaneous corpus cavernosal abscess. Case Rep Urol. 2013;2013:576146.

14. Paladino JR, Nascimento FJ, Gromatsky C, Pompeo ACL. Corpus cavernosum abscess after Winter procedure performance. BMJ Case Rep. 2014;2014:bcr2013202089.

15. Minami T, Kajikawa H, Kataoka K. Abscess of corpus cavernosum: two case reports. Hinyokika Kiyo. 2006;52:387-389 (in Japanese).

16. Ehara H, Kojima K, Hagiwara N, Phuoc NB, Deguchi T. Abscess of the corpus cavernosum. Int $J$ Infect Dis. 2007;11:553-554.

17. Koyama J, Namiki S, Kamiyama Y, et al. Total penectomy for corpus cavernosum abscess: a case report. Hinyokika Kiyo. 2015;61:109-114 (in Japanese).

18. Sater AA, Vandendris M. Abscess of corpus cavernosum. J Urol. 1989;141:949.

19. Kropman RF, de la Fuente RB, Venema PL, van Imhoff WL. Treatment of corpus cavernosum abscess by aspiration and intravenous antibiotics. J Urol. 1993;150:1502-1503. 